\title{
Universitätsbibliothek der Humboldt-Universität zu Berlin - Zentralbibliothek Naturwissenschaften
}

Die Humboldt-Universität befindet sich seit ihrer Gründung im Jahr 1810 im Zentrum von Berlin. Nach der Wiedervereinigung wurde schnell deutlich, dass ihre räumliche Situation in Berlin-Mitte keine ausreichende Möglichkeit für den dringend notwendigen Ausbau der mathematisch-naturwissenschaftlichen Fakultäten bot. So wurde bereits 1991 beschlossen, diesen Teil der Universität nach Berlin-Adlershof zu verlagern. Die Wahl fiel auf Adlershof, weil hier - den Absichten des Berliner Senates folgend - bis 2010 ein Wissenschafts- und Technologiepark entstehen soll, der schon heute über 360 technologieorientierte Unternehmen sowie über zehn auBeruniversitäre Forschungseinrichtungen beherbergt ${ }^{1}$. Die Institute für Chemie, Informatik und Mathematik der Humboldt-Universität sind bereits 2001 auf den sogenannten Campus Adlershof gezogen. Bis zum Jahr 2007 werden die anderen naturwissenschaftlichen Institute der Universität folgen ${ }^{2}$. Als eine der wichtigsten Infrastruktureinrichtungen wird das nach Erwin Schrödinger benannte Informations- und Kommunikationszentrum betrachtet, das die neue Zentralbibliothek für Naturwissenschaften sowie das Rechenzentrum der Universität beherbergen wird.

„Eine moderne Bibliothek wird ohne enge Kooperation mit Rechenzentrums- und Medieneinrichtungen nicht lebensfähig sein. ${ }^{\text {"3 }}$ Dieser Gedanke hat den Neubau der Zentralbibliothek für Naturwissenschaften auf dem Campus Adlershof von Anfang an begleitet. In einem Workshop mit dem Titel „Die Bibliothek der Zukunft" wurden erste Überlegungen zusammengetragen und diskutiert. Die für die Bauplanung wichtigen Grundsätze möchte ich hier gern noch einmal aufführen:

- Die Zusammenarbeit zwischen Bibliothek und Rechenzentrum wird durch ein gemeinsam genutztes Gebäude unterstützt.

- Im Mittelpunkt der Arbeit steht das Angebot zeitgemäßer Dienstleistungen.

- Bibliothek und Rechenzentrum werden den Umgang mit neuen Medien insbesondere durch Schulungen fördern.

- Die Bibliothek soll nicht nur der Informationsbeschaffung,

\begin{tabular}{|l|l|}
\hline Aufgaben der Bibliothek & Aufgaben des Rechenzentrums \\
\hline $\begin{array}{l}\text { Betreiber von File- und } \\
\text { Archivdiensten }\end{array}$ & $\begin{array}{l}\text { Betreiber der Kommunikations- } \\
\text { infrastruktur }\end{array}$ \\
\hline $\begin{array}{l}\text { Vermittlung der Kenntnisse im } \\
\text { Umgang mit elektronischen } \\
\text { Medien }\end{array}$ & $\begin{array}{l}\text { Organisation des Zugangs zu } \\
\text { elektronischen Medien }\end{array}$ \\
\hline $\begin{array}{l}\text { Garant für den freien Zugang zu } \\
\text { Informationen }\end{array}$ & $\begin{array}{l}\text { Koordinator des Zugangs } \\
\text { zu elektronischen } \\
\text { Informationsquellen }\end{array}$ \\
\hline $\begin{array}{l}\text { Anbieter von Informationen (z.B. } \\
\text { elektronisches Publizieren) }\end{array}$ & $\begin{array}{l}\text { Anbieter von elektronischen } \\
\text { Informationssystemen }\end{array}$ \\
\hline
\end{tabular}

Die gemeinsamen Aufgaben von Bibliothek und Rechenzentrum finden ihren Ausdruck auch auf administrativem und rechtlichem Gebiet: Beide Einrichtungen haben eine gemeinsame Benutzungs- und Gebührenordnung erarbeitet, die nicht nur im Erwin SchrödingerZentrum, sondern auch in allen anderen Einrichtungen der HumboldtUniversität gelten wird.

Alle beschriebenen Ideen sind in Bedarfsprogramm und Bauplanung eingeflossen, die für die Zentralbibliothek federführend Dr. Norbert Martin erarbeitet hat. Die Ausschreibung für einen begrenzt offenen Realisierungswettbewerb zum Informations- und Kommunikationszentrum erfolgte $1998^{4}$. Daniel Gössler aus Berlin gewann den Wettbewerb, weil sein Entwurf die anspruchsvollen Anforderungen am überzeugendsten erfüllte. Seine Konzeption berücksichtigte die verschiedenen Funktionen, die das Zentrum bietet. Seine besondere Leistung liegt vor allem in der Erhaltung bereits vorhandener Gebäude und der Einbindung dieser baulichen Substanz in das neue Nutzungskonzept. Große Teile alter Werkhallen konnten erhalten werden, so dass die Geschichte des Standortes für aufmerksame Besucher spürbar wird.

Die äußere Wirkung des Gebäudes bestimmt eine anthrazitfarbene Zinkfassade (Abb. 1). Über den Haupteingang erreicht man das 80

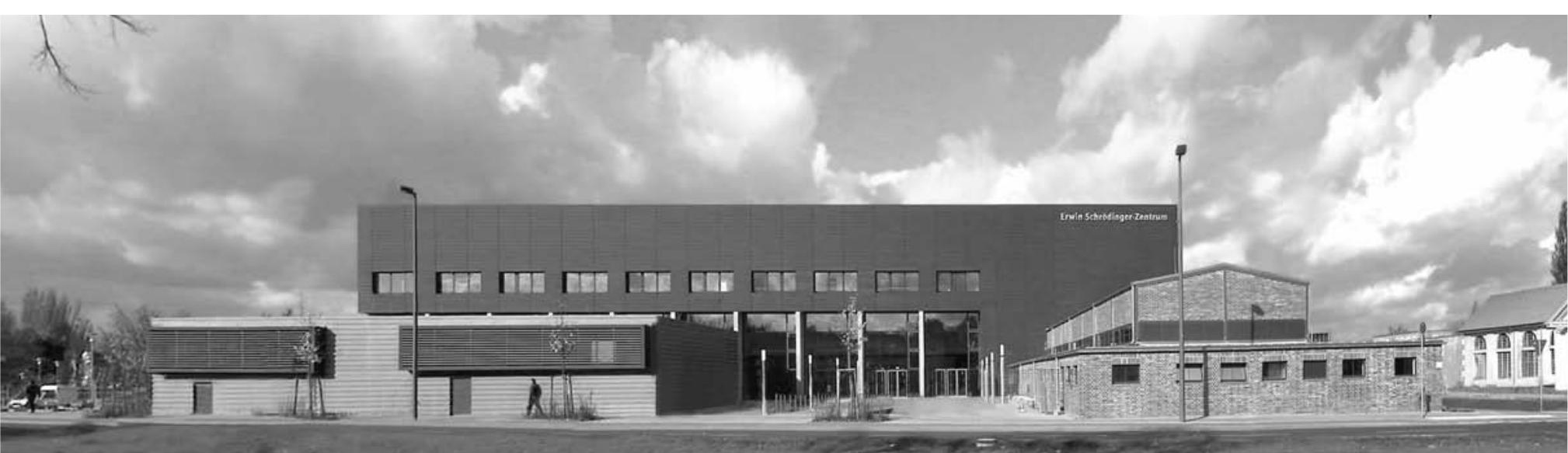

Abb. 1: Vorderansicht des Erwin Schrödinger-Zentrums

-speicherung und -lieferung dienen, sondern auch Begegnungsund Kommunikationsstätte sein.

- Die Bibliothek bzw. das Informations- und Kommunikationszentrum unterstützt die Zusammenarbeit mit außeruniversitären Forschungsinstituten und Wirtschaftsunternehmen und verspricht sich davon positive Effekte für Studium, Forschung, Lehre, Entwicklung und Produktion.

Aus diesen Vorgaben lassen sich die ganz konkreten Aufgaben von Bibliothek und Rechenzentrum ableiten:
Informationen zum Wissenschafts- und Technologiepark Adlershof finden Sie unter $<$ http://www.wista.de $>$.

Eine aktuelle chronologische Abfolge aller Ereignisse rund um die Entstehung des Campus Adlershof finden Sie unter $<$ http://www.huberlin.de/hu/adlershof/chron.html $>$.

3 Milan Bulaty, Michael Müller-Preußker, Peter Schirmbacher: Informationsund Kommunikationszentrum in Adlershof. In: Bibliothek. Forschung und Praxis 20 (1996) 2, S.257.

4 Senatsverwaltung für Bauen, Wohnen und Verkehr: Informationsund Kommunikationszentrum Adlershof. Begrenzt offener Realisierungswettbewerb. Ausschreibung. Berlin, im September 1998. 
Meter lange und 8 Meter hohe Foyer. Von hier aus gelangt man zur Bibliothek, zum PC-Pool, zu einem Konferenzsaal mit 160 Plätzen sowie zu einem kleinen Buchladen. Die erhaltene Portalwand einer alten Industriehalle erinnert kunsthistorisch Interessierte an sakrale Bauten. Ihr gegenüber am anderen Ende des Foyers sind zwei große Hörsäle gelegen.

Die bibliothekarische Idee von einem zentralen Lesesaal als Herz der Bibliothek mit einem großen Freihandbestand wird räumlich und gestalterisch einfallsreich umgesetzt. Der Lesesaal, wo Ruhe und eine angenehme Atmosphäre wesentlich sind für das konzentrierte Arbeiten, ist im Zentrum des Neubaus untergebracht. Er ist quadratisch (36 Meter x 36 Meter) und um 1,25 Meter abgesenkt. Dadurch sind Orientierung und Übersichtlichkeit auf einfache und deutliche Weise schon auf den ersten Blick erkennbar. Der Raum wird durch nordorientierte Oberlichter beleuchtet, die eine blendfreie Tagesbeleuchtung garantieren. Die Arbeitstische können in den Abendstunden mit zusätzlichen Lampen beleuchtet werden. Um Konzentration und Ruhe zu gewährleisten, sind Möbel und Wandverkleidung aus Holz gefertigt. Der Boden ist mit einem weinroten Teppich bedeckt, so dass eine warme und schallgedämpfte Atmosphäre entsteht.

Die Mitarbeiterräume der Bibliothek und des Rechenzentrums befinden sich im Obergeschoss des Neubaus. Das Magazin mit einer Kompaktanlage ist im Untergeschoss untergebracht. Der PC-Pool

\section{Kerndatenübersicht}

1. Name und Adresse

1.1 Universitätsbibliothek der Humboldt-Universität zu Berlin - Zentralbibliothek Naturwissenschaften

1.2 Rudower Chaussee 26.

1.3 D-12489 Berlin

1.4 Tel.: (030) 20 93-31 63

Fax (030) 20 93-54 04

E-Mailnawi@ub.hu-berlin.de

\section{Eingeschriebene Benutzer}

Gesamtzahl:

\section{Kapazitäten (vor dem Bau)}

3.1 Gesamtfläche $3500 \mathrm{~m}^{2}$

$\begin{array}{ll}3.2 \text { Benutzung } & 2130 \mathrm{~m}^{2}\end{array}$

3.3 Verwaltung $290 \mathrm{~m}^{2}$

3.4 Magazinfläche (inklusiv Freihand) $\quad 1080 \mathrm{~m}^{2}$

$\begin{array}{ll}3.5 & \text { Bände insgesamt } 388900\end{array}$

3.6 Bände Freihand 392850

3.7 Bände Magazine 96050

3.8 Leserplätze (einschl. Katalog) 256

3.8.1 vernetzt 31

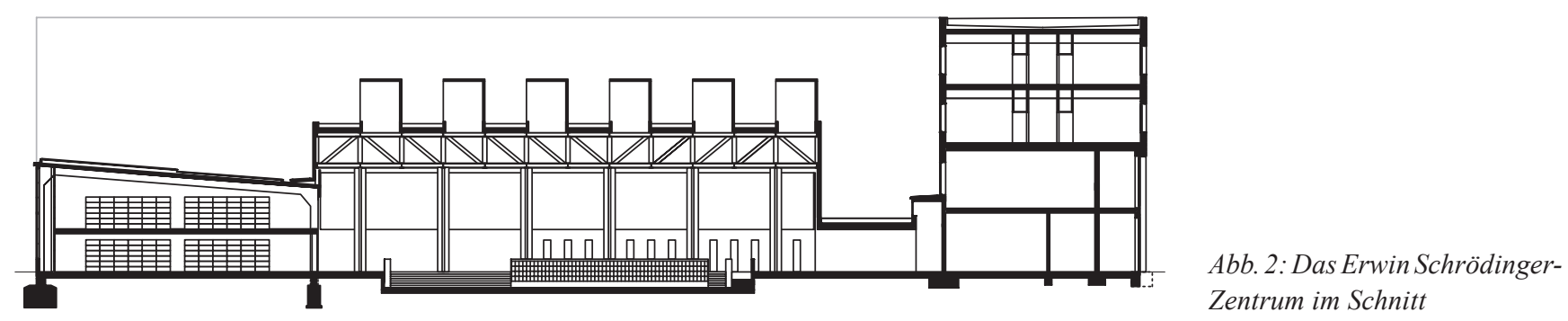

liegt in räumlicher Nähe zum Lesesaal; beide Räume sind durch Blickachsen miteinander verbunden. Neben der gemeinsamen Nutzung von Schulungs- und Gruppenräumen wird auch durch derartige, fast spielerische Effekte die Gemeinschaft von Bibliothek und Rechenzentrum unterstrichen und hervorgehoben.

Soweit es sich momentan einschätzen lässt, ist durch die Einweihung des Erwin Schrödinger-Zentrums im Frühjahr 2003 ein interessanter Ort in Berlin-Adlershof entstanden, der das Miteinander von Wissenschaft und Wirtschaft fördern wird. Die Zentralbibliothek für Naturwissenschaften und das Rechenzentrum, das sich fortan Computer- und Medienservice nennt wird, werden ihr Wissen und ihr Leistungsvermögen professionell einbringen.

\section{Anschrift des Autors:}

Dr. Milan Bulaty

Universitätsbibliothek der Humboldt-Universität zu Berlin D-10099 Berlin

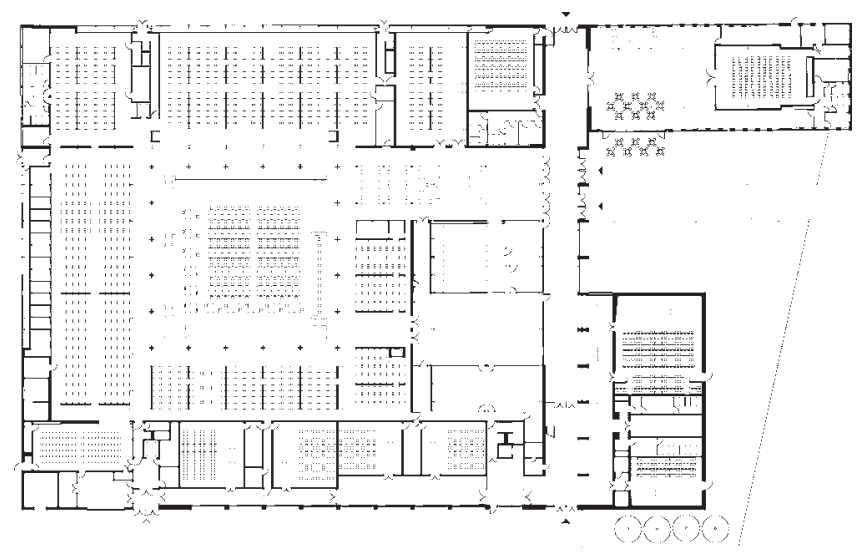




\subsection{2 nicht vernetzt}

4. Das Projekt

4.1 Typ: Neubau

4.2 Architekt(en), Firma

4.2.1 Architekten Gössler

4.2.2 Lehrter Str. 57, Haus 1, D-10557 Berlin

4.3 Beginn Bauarbeiten

4.4 Ende Bauarbeiten Januar

4.5 Inbetriebnahme
$225 \quad 4.6 \quad$ Gesamtfläche

4.7 Benutzung (inklusiv Freihand)

4.8 Verwaltung

4.9 Geschlossene Magazine $582 \mathrm{~m}^{2}$

4.10 Bände insgesamt $\quad 808000$

4.11 Bände Freihand 540000

4.12 Bände Magazine 268000

$2000 \quad 4.13$ Leserplätze 287

$\begin{array}{lll}2003 & 4.13 .1 \text { vernetzt } & 287\end{array}$

1.04 .2003 (erste Stufe)

1.11.2003 (zweite Stufe)

ca. 2007 (dritte Stufe)

5. Kapazitäten (nach dem Bau) - siehe unter 4 . 